\title{
Empirical Mode Decomposition (EMD) Based Denoising Method for Heart Sound Signal and Its Performance Analysis
}

\author{
Amy H. Salman, Nur Ahmadi, Richard Mengko, Armein Z. R. Langi, and Tati L. R. Mengko \\ School of Electrical Engineering and Informatics, Institut Teknologi Bandung
}

\begin{tabular}{l}
\hline Article Info \\
\hline Article history: \\
Received May 25, 2016 \\
Revised July 11, 2016 \\
Accepted July 27, 2016 \\
\hline Keyword: \\
Heart Sound \\
Denoising \\
Empirical Mode Decomposition
\end{tabular}

\begin{abstract}
In this paper, a denoising method for heart sound signal based on empirical mode decomposition (EMD) is proposed. To evaluate the performance of the proposed method, extensive simulations are performed using synthetic normal and abnormal heart sound data corrupted with white, colored, exponential and alpha-stable noise under different SNR input values. The performance is evaluated in terms of signal-to-noise ratio (SNR), root mean square error (RMSE), and percent root mean square difference (PRD), and compared with wavelet transform (WT) and total variation (TV) denoising methods. The simulation results show that the proposed method outperforms two other methods in removing three types of noises.
\end{abstract}

Copyright (c) 2016 Institute of Advanced Engineering and Science. All rights reserved.

\section{Corresponding Author:}

Amy H. Salman

School of Electrical Engineering and Informatics

Bandung Institute of Technology

Jl. Ganesha No 10, Bandung 40132, Indonesia

Email: amy@stei.itb.ac.id

\section{INTRODUCTION}

Cardiovascular disease (CVD) has long been the leading cause of death throughout the world with an estimate of 17.3 million people died in 2008 and is predicted to reach 23.3 million in 2030 [1]. According to WHO report, more than three quarters of the death takes place in low- and middle-income countries [2]. A low-cost and non-invasive diagnosis system based on heart sound can be used to minimize the risk of patients going into severe condition and reduce the financial burden through an early accurate diagnosis followed by appropriate treatment. This electronic auscultation technique utilizes advanced signal processing with fast computation capability, thanks to the advancement of computer technology.

However, to produce an accurate diagnosis result is not an easy task since, in practice, heart sound signal is always contaminated with noise and interference from various sources such as background noise, power interference, breathing or lung sounds, and skin movements in the surrounding environment. Thus, signal denosing method is of paramount importance to remove all these unwanted noise. A poor signal denoising method can lead to catastrophic result.

The most widely used method for denoising heart sound signal is based on wavelet transform (WT) [3-5], a powerful signal analysis tool with the ability to represent a signal simultaneously in the time and frequency. Despite the fact that the wavelet based denoising method has been proven to be able to provide good denoising performance, however, it suffers from several limitations. It requires predefined basis function selection (from too many choices) suited to signal under consideration, which limits the flexibility of the method. In addition, the decomposition level and thresholding technique of wavelet denosing also need to be carefully considered. Failing to choose the right decomposition level and thresholding technique will result in bad denoising performance. Varghees and Ramachandran employed another alternative method based on Total Variation (TV) [6]. TV method has been mostly used for image denoising due to its great benefit of preserving and enhancing important features such as edge in images. Even though it can be used for denoising 1D signal, nevertheless, there are very few literatures exploiting TV method for deonising heart sound. The highly non-stationary property of heart sound signal is not suitable to the nature of TV method which performs best on piecewise constant signals [7]. In addition, Figueiredo et. al. mentioned that the performance of TV 
method could produce better result than older wavelet based methods, but it was outperformed by recent state-the-art wavelet based methods [8].

Empirical mode decomposition (EMD), a relatively new non-linear and non-stationary signal analysis method [9], offers interesting feature of adaptive and data-driven decomposition capability. Since its inception, EMD has attracted many researchers around the globe to utilize it as denoising method [10-12]. However, the mechanism of discriminating the noise and useful information within decomposed signal and fitting it to heart sound signal to get good signal reconstruction performance remains challenging.

In this paper, we propose an EMD based denoising method for heart sound signals. To measure the performance of our proposed method, we perform repeated simulation over normal and abnormal synthetic heart sound signal burried under different types of noise with SNR input values ranging from 0 to $15 \mathrm{~dB}$. The qualitative evaluation is performed by visual inspection while quantitative evaluation is carried out by using three standard metrics: signal-to-noise ratio (SNR), root mean square error (RMSE), and percent root mean square difference (PRD).

The rest of this paper is organized as follows. Section 2. describes EMD denoising method with brief introduction to its theoritical background and mathematical notation. Section 3. explains the imulation setting and data. The simulation results and performance analysis of both qualitative and quantitative are given in Section 4 . Finally the conclusion is drawn in Section 5.

\section{EMD DENOISING METHOD}

\subsection{Empirical Mode Decomposition (EMD)}

Empirical mode decomposition (EMD), since firstly proposed by Huang in 1998 [9], has gained popularity as data analysis method especially for non-stationary and non-linear signals such as biomedical (including heart sound) signals. EMD, in contrast to other methods such wavelets and fourier which require predefined basis function, is fully data-driven method that does not require any a priori known basis. EMD adaptively decomposes a signal into a series of simple oscillatory AM-FM components called as intrinsic mode functions (IMFs) through iterative procedure (known as sifting). An IMF is defined as a function that satisfies two conditions: the number of extrema (maxima and minima) and zero crossing must be equal or differ by at most 1; and the average value of the envelopes derived from local maxima and minima is (approximately) zero.

Despite EMD still being lack of a solid mathematical foundation which could be used for theoritical analysis and performance evaluation, it has been proven to provide interesting and useful results. The sifting procedure of EMD for decomposing the signal $x(n)$ into IMFs is systematically described as follows:

1. Specify all the local extrema (maxima and minima) of $x(n)$

2. Interpolate between local maxima using cubic spline line to form upper envelope $e_{\max }(n)$ and local minima to form lower envelope $e_{\min }(n)$

3. Calculate the local mean based on formed upper and lower envelopes, $m(n)=\left(e_{\max }(n)+e_{\min }(n)\right) / 2$

4. Substract this mean from the original signal to extract the detail $d(n)=x(n)-m(n)$. If $d(n)$ does not satisfy IMF conditions (stopping criteria), the procedure 1) to 4) are iterated with new input signal $d(n)$

5. If $d(n)$ satisfies the criteria of an IMF, it is stored as an IMF, $h^{i}(n)=d(n)$ where $i$ refers to $i$ th IMF. Residue signal is obtained by substracting the IMF from the original signal, $r(n)=x(n)-h^{i}(n)$

6. Perform the same step from 1) with the new signal $r(n)$ until the final residue signal is constant or monotonic function. as follows:

After the completion of EMD process, the original signal can be written in terms of its IMF and residue signal

$$
x(n)=\sum_{i=1}^{L-1} h^{i}(n)+r^{L}(n)
$$

where $L$ refers to decomposition level and $i$ denotes IMF order. Lower-order of IMFs contains fast oscillation modes (high frequency) while higher-order of IMFs represent slow oscillation modes (low frequency).

\subsection{EMD Denoising}

EMD, whose decomposition is based on elementary substractions, enables perfect reconstruction of a signal. The EMD denoising method starts by identifying which IMFs carry dominantly noise and which IMFs contain primarily useful information. This is done by comparing the actual energy density with the estimated energy density (to 
form noise-only model [10]) of IMFs. The actual energy density of IMFs is calculated as follows

$$
E_{i}=\frac{1}{N} \sum_{n=1}^{N} h^{i}(n), \quad i=1,2,3, \cdots L
$$

with $i$ corresponds to IMF order. The estimated energy density (variance) of IMFs can be approximated using the formula [13] below

$$
\begin{gathered}
V_{1}=\left(\frac{\operatorname{median}\left(\left|h^{1}-\operatorname{median}\left(h^{1}\right)\right|\right)}{0.6745}\right)^{2} \\
V_{i}=\frac{V_{1}}{\beta} \rho^{-j}, \quad i=2,3,4, \cdots L
\end{gathered}
$$

where $\beta$ and $\rho$ equal to 0.719 and 2.01, respectively [10]. The IMFs whose actual energy density exceed the value of their estimated energy density defined by noise-only model are categorized as information-dominated signal and should be included in signal reconstruction step; otherwise those IMFs will be excluded.

Due to the fact that the noise embedded in IMFs is colored (not Gaussian distributed), even the informationdominated IMFs still may contain noise having different energy density. To remove those colored noise, IMFdependent threshold value is required. Considering that IMFs have zero mean and in any interval of zero crossing $\left[z_{j}^{i} z_{j+1}^{i}\right]$ the absolute amplitude of $i$ th IMF is very small, the thresholding scheme will be based on the single extrema $h^{i}\left(r_{j}^{i}\right)$, where $r_{j}^{i}$ corresponds to the extrema's time instance on this interval. The tresholding scheme which follows the hard thresholding is expressed as follows

$$
\tilde{h}^{i}\left(\left[z_{j}^{i} z_{j+1}^{i}\right]\right)= \begin{cases}h^{i}\left(\left[z_{j}^{i} z_{j+1}^{i}\right]\right), & \left|h^{i}\left(r_{j}^{i}\right)\right|>T_{i} \\ 0, & \left|h^{i}\left(r_{j}^{i}\right)\right| \leq T_{i}\end{cases}
$$

where $h^{i}\left(\left[z_{j}^{i} z_{j+1}^{i}\right]\right)$ represents the samples from time instant $z_{j}^{i}$ to $z_{j+1}^{i}$ of $t$ th IMF. The threshold value used in this scheme is expressed below

$$
T_{i}=C \sqrt{V_{i} 2 \ln N}, \quad i=1,2,3, \cdots L
$$

where $C$ is 0.1 found by empirical simulations and $V_{i}$ is estimated energy density (variance) of $i$ th IMF. This thresholding scheme which is inspired and adapted from wavelet [11] will set to zero all the samples from time instant $\left[z_{j}^{i} z_{j+1}^{i}\right]$ if the single extrema amplitude below the theshold value meaning that there is no useful information (only noise) in the specified time instant. Otherwise, all the samples will be retained.

The final signal reconstruction can be obtained by summing up all the included IMFs (whose actual energy density exceeding its estimated estimated energy as described previously) using the following formula

$$
\hat{y}=\sum_{i=p}^{q} \tilde{h}(n)
$$

where $p$ and $q$ indicates the lowest and highest index of included IMF.

\section{SIMULATION SETTING}

To evaluate the qualitative and quantitative performance of our proposed method, we performed repeated simulations using synthetic heart sound data obtained from University of Michigan's Heart Sound \& Murmur Library [14]. In this simulations, three types of heart sound signals used for simulations and their respective recording location are 'Normal S1 S2' (Apex, Supine, Bell), 'S3 Gallop' (Apex, Left Ducubitus, Bell) and 'S4 Gallop' (Apex, Left Ducubitus, Bell). These data are encoded in 44,100 Hz sample rate, 16 bits/sample, and 1 minutes of data length. The data was then down-sampled into $2000 \mathrm{~Hz}$ to increase the computation process without violating Nyquist theorem, since the frequency content of heart sound data is maximum at around $700 \mathrm{~Hz}$. The simulations were carried out 100 times in each case using MATLAB 2015a which runs on Intel(R) Core(TM) i7-4790 CPU @ 3.6 GHz Windows 7 environmfßtmeasure the performance under various noises, we added four types of noises, which are white, colored (brown), exponential and alpha-stable noise to the clean input signal $y(n)$ to form noisy signal $x(n)$. In each noise case, we used different input SNR level of $0,5,10$, and $15 \mathrm{~dB}$. The noisy heart sound signal, $x(n)$, is expressed as

$$
x(n)=y(n)+e(n), \quad n=0,1,2, \cdots N-1
$$

where $y(n)$ and $e(n)$ denotes the clean signal and noise, respectively. 
For the purpose of performance benchmark, we also performed simulations over the same data using Wavelet Transform (WT) and Total Variation (TV) based denoising methods. In WT based simulation, Daubechies db10 wavelet function was used since it highly resembles the heart sound signal, which lead to yield better performance. In addition, db10 wavelet has orthogonal property which enables perfect reconstruction of signal and have been reported to produce best result among others [3]. The decomposition level, $N=5$, is chosen as recommended in [4]. Hard thresholding technique is selected as it provides better result compared to soft thresholding technique. MATLAB has built-in function wden for wavelet denoising as described in [15]. Several input parameters and their setting for this function are explained in Table 1 [16]. Parameter rigsure represents the selection using the principle of Steins Unbiased Risk Estimate (SURE), h means hard thresholding, and $\mathrm{m} \mathrm{n}$ denotes threshold rescaling using a level-dependent estimation of the level noise.

Table 1. Input parameters setting

\begin{tabular}{lll}
\hline Parameter & \multicolumn{1}{c}{ Description } & Chosen Setting \\
\hline $\mathrm{s}$ & original signal & $x(n)$ \\
tptr & threshold selection rule & rigsure \\
sorh & thresholding technique & $\mathrm{h}$ \\
scal & threshold's rescaling method & $\mathrm{mln}$ \\
$\mathrm{n}$ & decomposition level & 5 \\
wav & (mother) wavelet function & $\mathrm{db} 10$ \\
\hline
\end{tabular}

As for TV based denoising method, a Majorization-Minimzation (MM) algorithm [7] was used to solve a sequence of optimization problems. The parameter $\lambda$ is set to 0.3 based on the experiment of and characteristic heart sound signals. The algorithm is run for 50 iterations to find more accurate result.

\section{RESULTS AND ANALYSIS}

Figure 1(a) and Figure 1(b) shows 'Normal S1 S2' heart sound signal $x(n)$ decomposition into 11 IMFs along with its final residue signal and the IMFs' energy density comparison under $0 \mathrm{~dB}$ level of white noise, respectively. As shown in Figure 1(b), only information-dominated IMFs (number 3, 4, 5, 6, 7, and 11) will be processed for final reconstruction, which leads to a term "partial reconstruction" of a signal.

The qualitative performance evaluation of our proposed method compared to other denoising methods were performed by visual inspection and comparison. Figure 2 presents the input clean signal, noisy signal, and denoised (reconstructed) signal of 'S4 Gallop' using wavelet, TV, and EMD denoising methods. In each noise case, only one simulation result under $5 \mathrm{~dB}$ input SNR level is shown. Based on Figure 2, it is shown that EMD denosing method performs better among others in three types of noises: white (a), brown (b) and exponential noise (c) as its denoised signal most resembles the original signal. If we look closely and zoom in the figure, we will know that the amplitude of denoised signal by TV method are slightly reduced. Even though the denoised signal by TV method keeps the amplitude of its main components almost the same as original one, the amplitude outside the main components interval

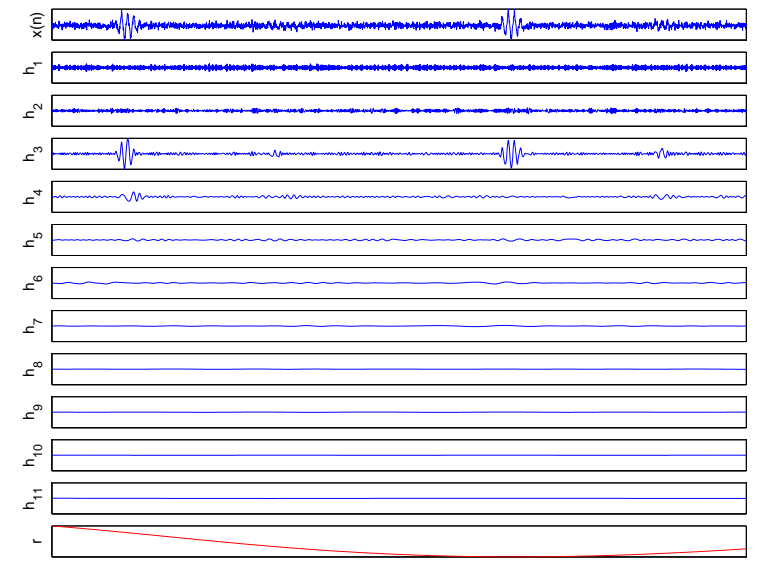

(a) EMD based signal decomposition

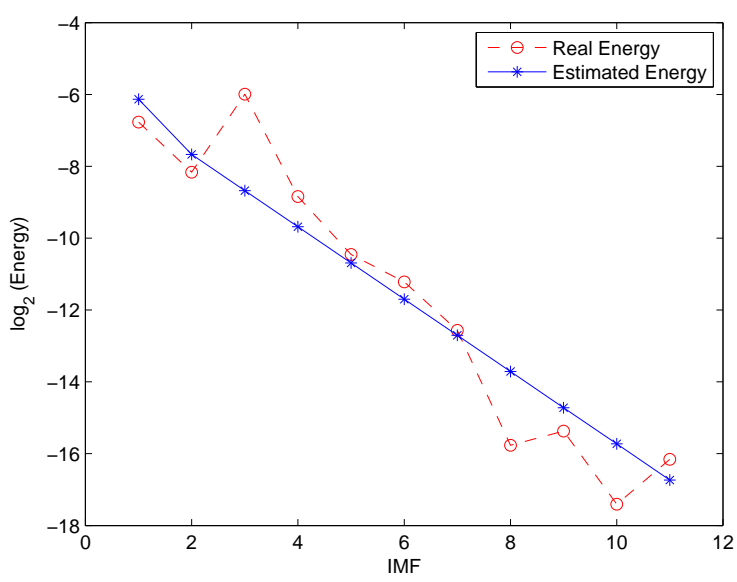

(b) Real vs estimated energy density of IMFs

Figure 1. Signal decomposition and energy density comparison of IMFs under $0 \mathrm{~dB}$ level of white noise 


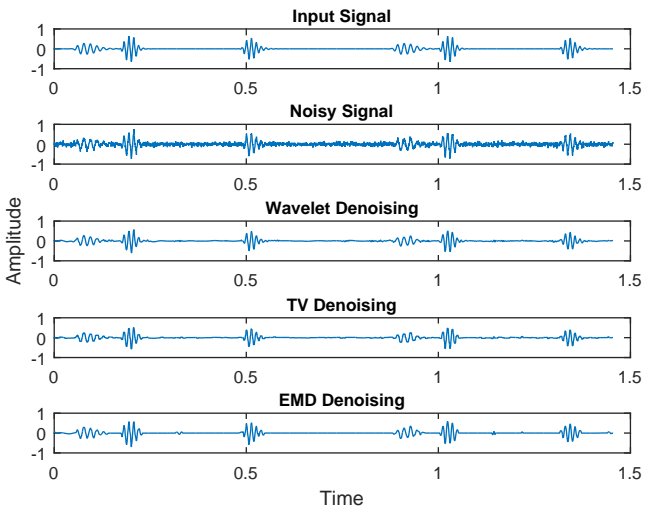

(a) White noise with SNR $5 \mathrm{~dB}$

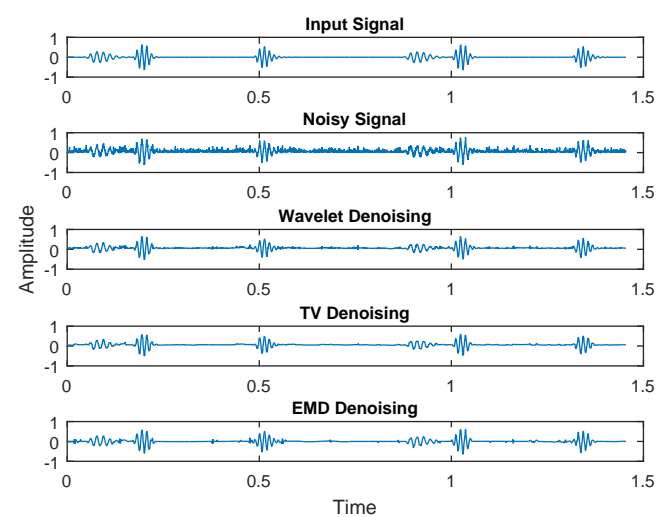

(c) Exponential noise with SNR $5 \mathrm{~dB}$

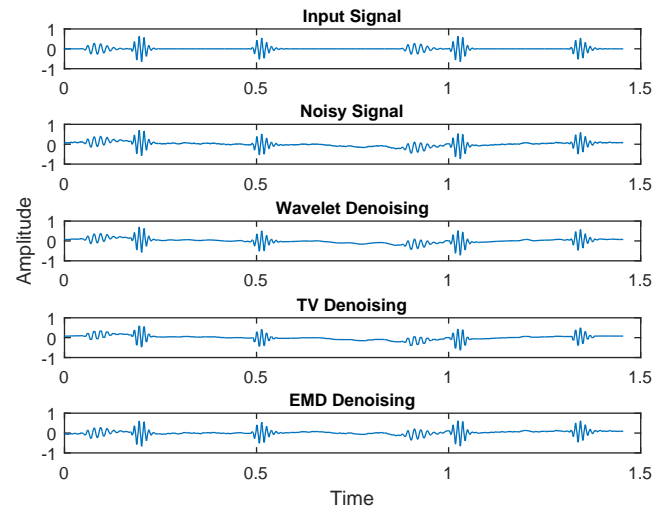

(b) Brown noise with SNR $5 \mathrm{~dB}$

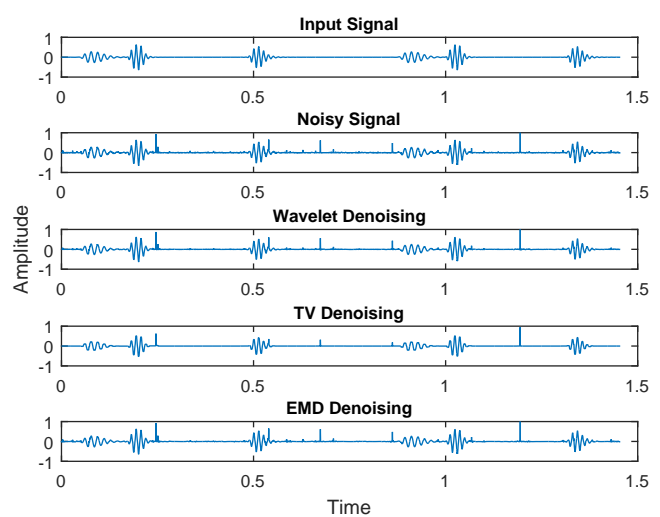

(d) Alpha-stable noise with SNR $5 \mathrm{~dB}$

Figure 2. Visual performance comparison of 'S4 Gallop' heart sound signal denoising methods

is slightly changed compared to the original signal. As for alpha-stable noise as shown in Figure 2(d), WT and TV denoising methods pefrorms better than EMD method.

In order to obtain more exact comparison, a quantitative performance was evaluated based on three metrics namely signal-to-noise ratio (SNR), root mean square error (RMSE), and percent root mean square difference (PRD),

Table 2. Performance comparison of denoising methods for 'S3 Gallop' heart sound data

\begin{tabular}{|c|c|c|c|c|c|c|c|c|c|c|}
\hline \multirow{2}{*}{$\begin{array}{l}\text { Noise } \\
\text { Type }\end{array}$} & \multirow{2}{*}{$\begin{array}{l}\text { Input } \\
\text { SNR }\end{array}$} & \multicolumn{3}{|c|}{ SNR (dB) } & \multicolumn{3}{|c|}{ RMSE } & \multicolumn{3}{|c|}{ PRD (\%) } \\
\hline & & WT & TV & EMD & WT & TV & EMD & WT & TV & EMD \\
\hline \multirow{4}{*}{ White } & 0 & 8.9463 & 7.6747 & 9.9222 & 0.0391 & 0.0452 & 0.0352 & 35.7720 & 41.3448 & 32.1339 \\
\hline & 5 & 12.7895 & 11.8554 & 13.2863 & 0.0251 & 0.0280 & 0.0240 & 22.9760 & 25.5490 & 21.9542 \\
\hline & 10 & 16.6698 & 13.6183 & 17.6169 & 0.0161 & 0.0228 & 0.0145 & 14.6864 & 20.8534 & 13.2708 \\
\hline & 15 & 20.5798 & 14.2784 & 20.9220 & 0.0102 & 0.0211 & 0.0101 & 9.3646 & 19.3253 & 9.3160 \\
\hline \multirow{4}{*}{ Brown } & 0 & -1.3465 & -1.3822 & $\mathbf{0 . 5 8 7 5 3}$ & 0.1329 & 0.1334 & 0.1071 & 121.4947 & 121.9054 & 97.8917 \\
\hline & 5 & 3.4123 & 3.1738 & 5.0755 & 0.0768 & 0.0786 & 0.0641 & 70.1732 & 71.8188 & $\mathbf{5 8 . 5 9 4 4}$ \\
\hline & 10 & 8.3727 & 7.5069 & 9.8494 & 0.0431 & 0.0471 & 0.0374 & 39.4378 & 43.0857 & 34.1929 \\
\hline & 15 & 13.1883 & 10.8689 & 15.1542 & 0.0246 & 0.0316 & 0.0205 & 22.499 & 28.8733 & 18.6951 \\
\hline \multirow{4}{*}{ Exponential } & 0 & -0.9209 & -0.8023 & 6.2413 & 0.1217 & 0.1200 & 0.0537 & 111.2174 & 109.7049 & 49.0467 \\
\hline & 5 & 4.0152 & 4.1953 & 10.1811 & 0.0689 & 0.0675 & 0.0349 & 63.0014 & 61.7036 & 31.8804 \\
\hline & 10 & 8.8459 & 8.4530 & 14.5646 & 0.0395 & 0.0413 & 0.0223 & 36.1247 & 37.7918 & 20.3669 \\
\hline & 15 & 13.6832 & 11.6200 & 18.3461 & 0.0226 & 0.0287 & 0.0157 & 20.6983 & 26.2448 & 14.3572 \\
\hline \multirow{4}{*}{ Alpha-stable } & 0 & 9.8326 & 10.1323 & 8.0622 & 0.0440 & 0.0423 & 0.0564 & 40.2600 & 38.6638 & 51.5371 \\
\hline & 5 & 15.7833 & 13.0523 & 11.3505 & 0.0220 & 0.0272 & 0.0392 & 20.1374 & 24.8517 & 35.8671 \\
\hline & 10 & 20.1803 & 13.8206 & 14.2772 & 0.0158 & 0.0259 & 0.0336 & 14.4564 & 23.6848 & 30.6898 \\
\hline & 15 & 25.2577 & 14.3523 & 18.3497 & 0.0073 & 0.0213 & 0.0262 & 6.6370 & 19.4375 & 23.9710 \\
\hline
\end{tabular}


which are calculated as follows:

$$
\begin{gathered}
S N R=10 \log _{10} \frac{\sum_{n=1}^{N}[y(n)]^{2}}{\sum_{n=1}^{N}[y(n)-\hat{y}(n)]^{2}} \\
R M S E=\sqrt{\frac{\sum_{n=1}^{N}[y(n)-\hat{y}(n)]^{2}}{N}} \\
P R D=\sqrt{\frac{\sum_{n=1}^{N}[y(n)-\hat{y}(n)]^{2}}{\sum_{n=1}^{N}[y(n)]^{2}}} \times 100
\end{gathered}
$$

where $y(n)$ denotes the clean original signal, $\hat{y}(n)$ refers to the denoised (reconstructed) signal, and $N$ represents the length of the signal.

SNR is defined as the ratio of the power of a signal (useful information) and the power of noise (irrelevant signal). RMSE is used to measure the accuracy of denoising method in preserving the quality of information in the denoised signal by calculating the sample standard deviation of the differences between denoised signal and original signal. PRD is frequently used as a method of quantifying the distortion or the difference between the original and the reconstructed signal. The PRD indicates reconstruction fidelity by point wise comparison with the original data. A denoising method is said to perfom better if at a particular input SNR, the value of output SNR is larger while the value of RMSE and PRD are smaller.

Comparative simulation results of three denoising methods (WT, TV, and EMD) over 'S3 Gallop' heart sound data on the basis of SNR, RMSE, and PRD are shown in Table 2. The simulation result values were rounded into 4 digits after comma. Highlighted (bold) values indicates the best performance among others. It is shown that for three cases of noises (white, brown, and exponential) under different input SNR values $(0,5,10$, and $15 \mathrm{~dB}$ ), EMD denoising method consistently yields largest SNR value, and smallest RMSE and PRD values (see bold values). For instance in white noisy environment with $0 \mathrm{~dB}$ input SNR level, EMD method shows SNR value $9.9222 \mathrm{~dB}$, RMSE 0.0352 and PRD $32.1339 \%$ where as WT (TV) method shows 8.9463 (7.6747) dB SNR, 0.0391 (0.0452) RMSE, and $35.7720 \%(41.3448 \%)$ PRD. The performance of EMD method in these three types of noises for other heart sound signals ('Normal S1 S2' and 'S4 gallop') over input SNR level range (0 dB - $15 \mathrm{~dB})$ is superior as well compared to WT and TV methods. However, for heart sound signals contaminated with alpha-stable noise, EMD method does not perform well compared to its counterparts especially for input SNR level 0 - $10 \mathrm{~dB}$. In this type of noise, on average, WT method outperforms other two methods, except for the case of $0 \mathrm{~dB}$ input SNR where TV method produces the best performance on all three metrics. Alpha-stable noise being used in this simulation represents the impulsive noise or disturbance characterized by high amplitude and short time duration within arbitrary location along the data. This impulsive disturbance usually occurs when there is quick movement or friction between chest skin and stethoscope during recording heart sound data. This alpha-stable noise has four parameters: $\alpha$ (characteristic exponent), $\beta$ (skewness), $\gamma$ (scale) and $\delta$ (location) [17]. Parameter $\alpha$ indicates the tail of distribution while $\beta$ specifies whether the distribution is right- or left-skewed. In this simulation, we used $\alpha=1.6, \beta=1, \gamma=0.1$ and $\delta=0$.

Graphical visualization of comparative simulation results of 'S4 Gallop' heart sound signal under four types of noises is depicted in Figure 3. Figure 3(a-c) shows the comparative output SNR, RMSE and PRD value of three denoising methods with respect to different input SNR levels in white noisy environment. It is shown that EMD method (blue line with triangle point) on avarege performs better than WT (black line with rectangle point) and TV (red line with circle point), indicated by larger output SNR value and smaller RMSE and PRD values. The same trend is also observed in simulation results over brown and exponential noisy signal as shown in Figure 3(d-f) and Figure 3(g-i). EMD is equivalent to dyadic filter structure which can effectively decompose fractional Gaussian noise processes such as white and colored (brown) noises. This leads to effective denoising method over different class of fractional Gaussian noises [18-20]. Moreover, EMD method does not require any predefined basis function and is fully data-driven which offers more flexibility and adaptability to any signal under consideration. However, EMD method does not perform well compared to its counterparts under alpha-stable noise simulation as shwon in Figure $3(\mathrm{j}-1)$. According to our observation during repeated simulations, we chose constant value $C=0.6$ in threshold value calculation within EMD denoising mechanism to obtain good performance. This constant value applies well on three types of noises (white, brown and exponential). However, based on our simulation, the performance of EMD denoising method under alpha-stable noise can be improved by increasing the constant value $C$ up to 1.5 . In addition, to mitigate this impulsive disturbance in heart sound analysis, an adaptive selection algorithm based on Heron's formula can be employed in the subsequent process [21]. 


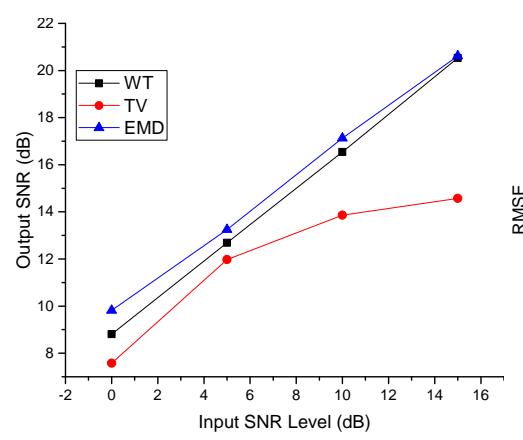

(a)

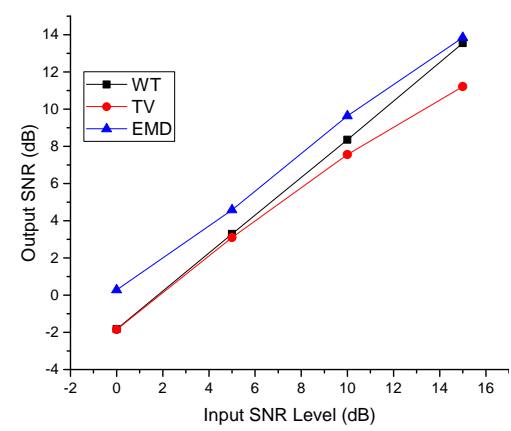

(d)

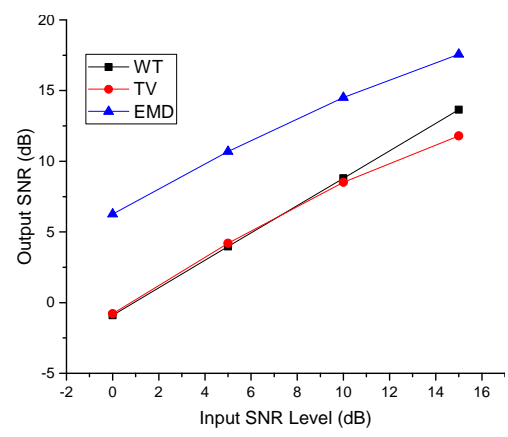

(g)

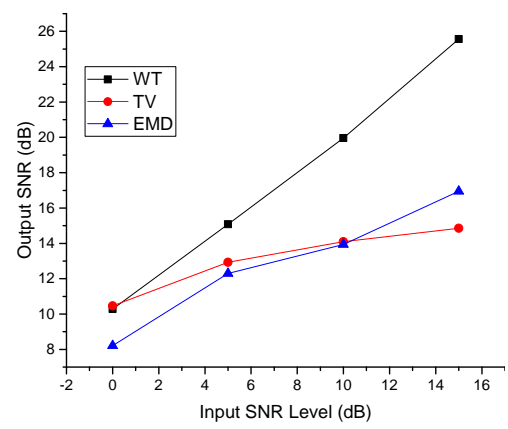

(j)

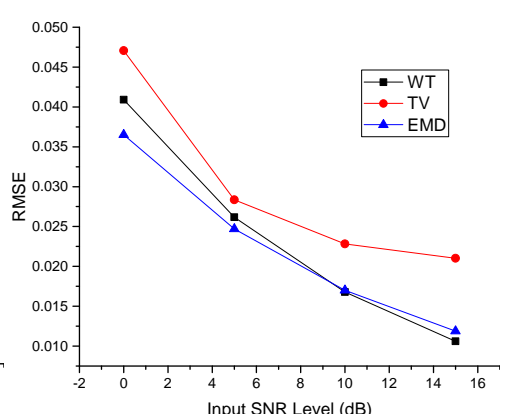

(b)

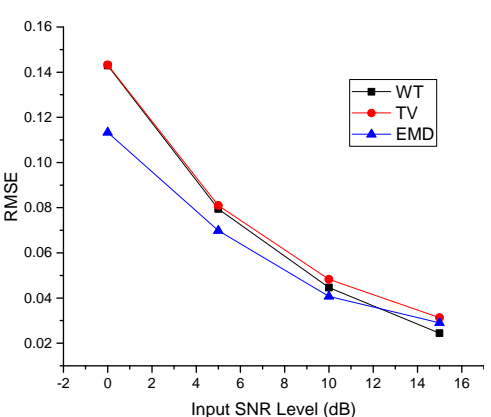

(e)

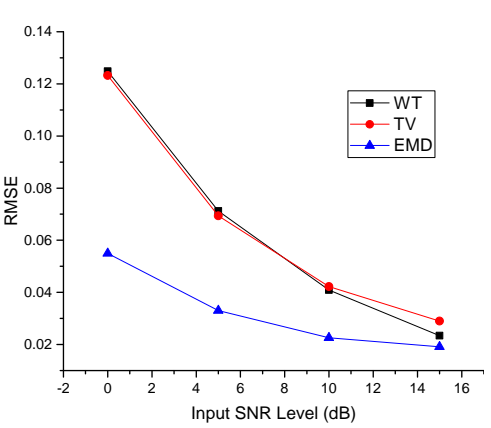

(h)

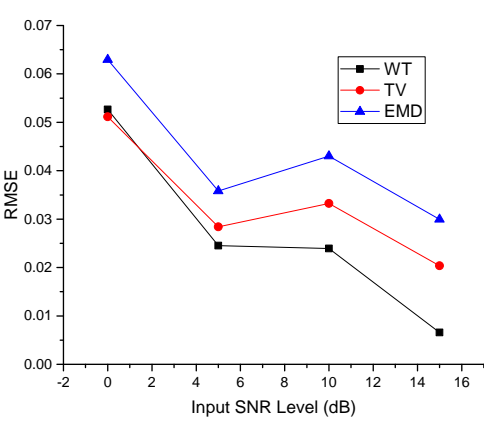

(k)

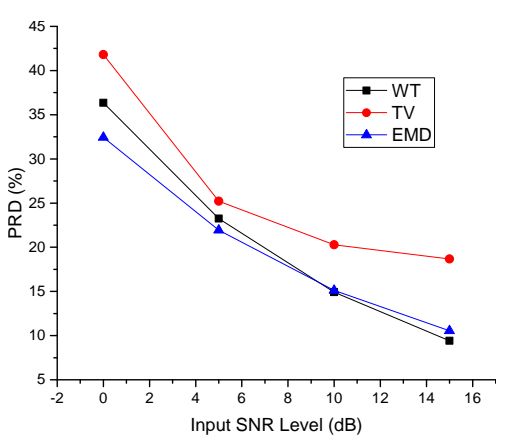

(c)

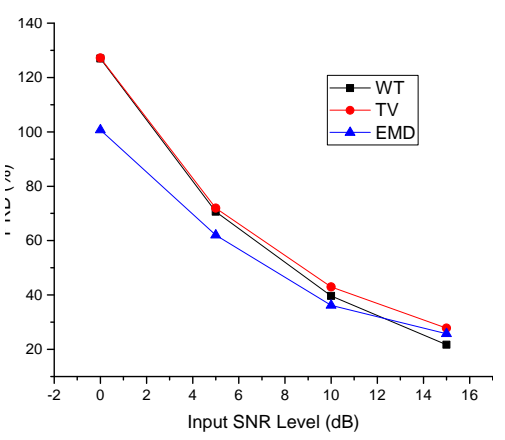

(f)

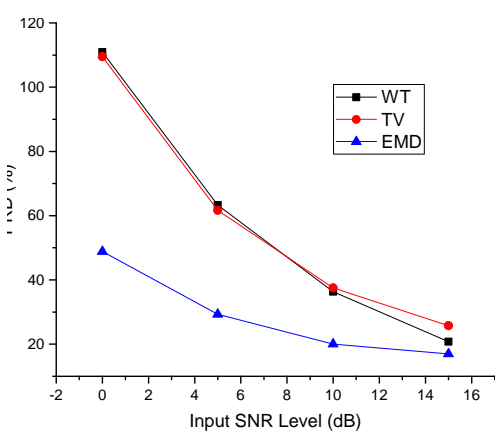

(i)

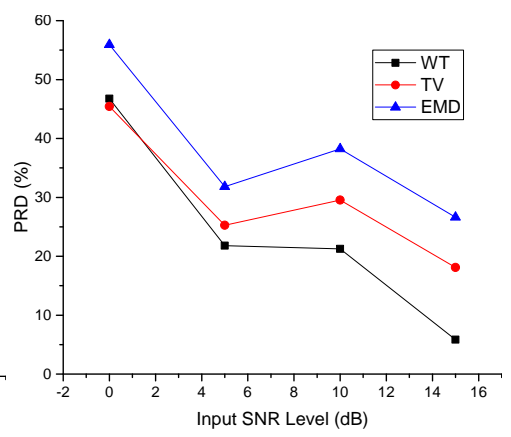

(1)

Figure 3. Performance comparison over 'S4 Gallop' heart sound signal under (a-c) white (d-f) brown (g-i) exponential and (j-1) alpha-stable noise 


\section{CONCLUSION}

Empirical Mode Decomposition (EMD) based denoising method is proposed in this paper. Its performance and analysis compared to other two methods based on wavelet transform (WT) and total variation (TV) are presented. Four types of noises with input SNR level $0 \mathrm{~dB}, 5 \mathrm{~dB}, 10 \mathrm{~dB}$ and $15 \mathrm{~dB}$ are artificially added to clean original normal and abnormal heart sound signals obtained from the University of Michigan Health System. Based on extensive simulations, our proposed EMD based denoising method consistently yields better performance in terms of three standard metrics: signal-to-noise ratio (SNR), root mean square error (RMSE), and percent root mean square difference (PRD) under white, colored (brown) and exponential noises. As for alpha-stable noise, on average, WT and TV based denoising methods perform better than EMD method.

\section{REFERENCES}

[1] G. Redlarski, D. Gradolewski, and A. Palkowski, "A system for heart sounds classification,” PloS one, vol. 9, no. 11, p. e112673, 2014.

[2] WHO, Global status report on noncommunicable diseases 2014. World Health Organization, 2014.

[3] L. H. Cherif, S. Debbal, and F. Bereksi-Reguig, "Choice of the wavelet analyzing in the phonocardiogram signal analysis using the discrete and the packet wavelet transform," Expert Systems with Applications, vol. 37, no. 2, pp. 913-918, 2010.

[4] S. R. Messer, J. Agzarian, and D. Abbott, "Optimal wavelet denoising for phonocardiograms," Microelectronics Journal, vol. 32, no. 12, pp. 931-941, 2001.

[5] F. Liu, Y. Wang, and Y. Wang, "Research and implementation of heart sound denoising," Physics Procedia, vol. 25, pp. 777-785, 2012.

[6] V. N. Varghees and K. Ramachandran, "A novel heart sound activity detection framework for automated heart sound analysis," Biomedical Signal Processing and Control, vol. 13, pp. 174-188, 2014.

[7] “Total variation denoising (an MM algorithm)," Technical Report, Polytechnic University, Mar. 2014.

[8] M. A. Figueiredo, J. B. Dias, J. P. Oliveira, and R. D. Nowak, "On total variation denoising: A new majorizationminimization algorithm and an experimental comparisonwith wavalet denoising," in 2006 IEEE International Conference on Image Processing. IEEE, 2006, pp. 2633-2636.

[9] N. E. Huang, Z. Shen, S. R. Long et al., "The empirical mode decomposition and the hilbert spectrum for nonlinear and non-stationary time series analysis," in Proceedings of the Royal Society of London A: Mathematical, Physical and Engineering Sciences, vol. 454, no. 1971. The Royal Society, 1998, pp. 903-995.

[10] G. Rilling, P. Flandrin, and P. Goncalves, "Detrending and denoising with empirical mode decomposition," in Proceedings of the European signal processing conference (EUSIPCO04), vol. 2, 2004, pp. 1581-1584.

[11] Y. Kopsinis and S. McLaughlin, "Development of EMD-based denoising methods inspired by wavelet thresholding," IEEE Transactions on Signal Processing, vol. 57, no. 4, pp. 1351-1362, 2009.

[12] M. A. Kabir and C. Shahnaz, "Denoising of ECG signals based on noise reduction algorithms in EMD and wavelet domains," Biomedical Signal Processing and Control, vol. 7, no. 5, pp. 481-489, 2012.

[13] W. Wu and H. Peng, "Application of EMD denoising approach in noisy blind source separation," Journal of Communications, vol. 9, no. 6, 2014.

[14] Heart Sound \& Murmur Library, University of Michigan Health System, Ann Arbor, MI. [Online]. Available: http://www.med.umich.edu/lrc/psb_open/repo/primer_heartsound/primer_heartsound.html

[15] M. Misiti, Y. Misiti, G. Oppenheim, and J.-M. Poggi, Wavelet Toolbox User's Guide, Rev. 4.14 (Rel. 2014b), The MathWorks, Inc., Oct. 2014.

[16] A. H. Salman, N. Ahmadi, R. Mengko, A. Z. R. Langi, and T. L. R. Mengko, "Performance comparison of denoising methods for heart sound signal," in 2015 International Symposium on Intelligent Signal Processing and Communication Systems (ISPACS). IEEE, 2015, pp. 435-440.

[17] M. Veillette, Alpha-Stable distributions, Boston University, Aug. 2015.

[18] Z. Wu and N. E. Huang, "A study of the characteristics of white noise using the empirical mode decomposition method," Proceedings of the Royal Society of London. Series A: Mathematical, Physical and Engineering Sciences, vol. 460, no. 2046, pp. 1597-1611, 2004.

[19] P. Flandrin and P. Goncalves, "Empirical mode decompositions as data-driven wavelet-like expansions," International Journal of Wavelets, Multiresolution and Information Processing, vol. 2, no. 04, pp. 477-496, 2004.

[20] P. Flandrin, P. Gonçalves, and G. Rilling, "EMD equivalent filter banks, from interpretation to applications," Hilbert-Huang Transform and Its Applications, pp. 57-74, 2005.

[21] A. H. Salman, N. Ahmadi, R. Mengko, A. Z. Langi, and T. L. Mengko, "Automatic segmentation and detection of heart sound components S1, S2, S3 and S4," in 4th International Conference on Instrumentation, Communications, Information Technology, and Biomedical Engineering (ICICI-BME 2015). IEEE, 2015, pp. 103-107. 